\title{
Diversidade da fauna edáfica de um latossolo vermelho-amarelo sob diferentes usos no cerrado piauiense
}

\author{
Juliana Vogado Coelho' ${ }^{1}$, Bruna de Freitas Iwata ${ }^{2}$, Tancio Gutier \\ Ailan Costa ${ }^{3}$, Laécio Miranda da Cunha ${ }^{4}$, Nayara Caroline Moreira \\ Leopoldo $^{5}$, Gleide Ellen dos Santos Clementino5, Ana Carla Ribeiro \\ Maciel $^{5}$
}

${ }_{1}^{1}$ Universidade Federal do Paraná. Programa de Pós-Graduação em Ciência do Solo. Rua dos Funcionários, 1540. Juvevê. Curitiba-PR, Brasil (CEP 80035-050). E-mail: vogado.juliana@gmail.com.

${ }^{2}$ Instituto Federal de Educação, Ciência e Tecnologia do Piauí. Campus Teresina Central. Praça da Liberdade, 1597. Centro. Teresina-PI, Brasil (CEP 64000-040).

${ }^{3}$ Universidade Federal do Ceará. Mestrado em Ciência do Solo. Campus Pici. Fortaleza-CE (CEP 60455-900).

4Instituto Federal de Educação Ciência e Tecnologia Goiano. Programa de PósGraduação em Agroquímica. Campus Rio Verde. Rodovia Sul Goiana, km 01. Zona Rural. Rio Verde-GO, Brasil (CEP 75901-970).

${ }^{5}$ Instituto Federal do Piauí. Curso de Tecnologia em Gestão Ambiental. Campus Corrente. Rua Projetada Trinta e Seis, 380. Nova Corrente. Corrente-PI, Brasil (CEP 64980-000).

Resumo: A qualidade do solo está relacionada com a capacidade funcionamento dentro do ecossistema, e a fauna edáfica sendo sensível, responde com relativa rapidez, ao impacto de diferentes tipos de sistemas de produção, possibilitando, o seu uso como componente ativo na avaliação como indicador biológico na qualidade do solo. 0 presente estudo objetivou avaliar a fauna edáfica em latossolo vermelho-amarelo sob sistema agroflorestal recém instalado e em monocultivo de soja no Cerrado piauiense. Em cada área foi demarcada uma parcela amostral de $10 \mathrm{~m} \mathrm{x}$ $10 \mathrm{~m}$. Para captura dos indivíduos da fauna edáfica, utilizou-se armadilhas do tipo pitfall, sendo distribuídas, aleatoriamente, 10 armadilhas em cada área. A coleta da amostragem e monitoramento das armadilhas foi realizada em um período de 60 dias em um ciclo de 15 dias, considerando o período de estiagem na região, no qual foram quantificadas e separadas em nível de ordem e caracterizada quanto à frequência, dominância e constância. 0 estudo permitiu a identificação de sete ordens, e coletados 849 indivíduos pertencente aos grupos taxonômicos Squamata, Diplopoda, Araneae, Coleoptera, Dermaptera, Hymenoptera, Orthoptera. Verificou-se a presença de maior infuencia da ordem Hymenoptera (620 indivíduos) sob o sistema agroflorestal, no qual a área sofre influencia direta das condições natural do ambiente o que caracteriza a preservação dos

Recebido:

20/05/2018

Aceito:

03/08/2018

Publicado:

31/08/2018

Acesso aberto

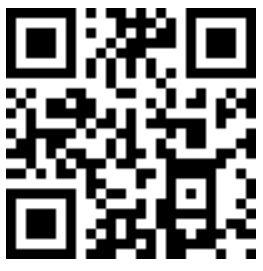

ORCID

(1) 0000-0003-2099-9997 Juliana Vogado Coelho 
indivíduos local. Na área sob o monocultivo da soja os indivíduos com maior representatividade foi a Coleoptera (121 indivíduos) o qual pode-se justificar por serem consideradas como pragas em sistemas agrícolas a depender da familia. As mudanças na caracterização da fauna edáfica foram observadas nos diferentes sistemas de uso com diversidade dos indivíduos coletados. Conclui-se que na área sob o sistema agroflorestal são mais estáveis em termos de biodiversidade da fauna edáfica, quando comparados com áreas agrícolas como do monocultivo da soja que sofre influência direta pelas práticas adotadas nos cultivos, provocando alteração da fauna edáfica.

Palavras-chave: Qualidade do solo; Biodiversidade edáfica; Biostema agroflorestal.

\begin{abstract}
Diversity of the edaphic fauna of a red-yellow latosol under different uses in the Piauí cerrado. The quality of the soil is related to the capacity of execution within the ecosystem, the fauna is a graphical representation, sensitive to the relative speed, to the impact of different types of production systems, making possible the use of components as an indicator in the quality of an indicator do it yourself. The present study aimed at an edaphic fauna in red-yellow latosol under the newly installed agroforestry system and in monoculture of non-phytosanitary soybean Cerrado. In each area a sampling plot of $10 \mathrm{~m} \times 10 \mathrm{~m}$ was demarcated. To capture the fauna, traps were used as traps, and 10 streaks were randomly distributed in each area. Sampling and trapping were carried out over a period of 60 days over a period of 15 days, considering the sampling period in the region, not being quantified and classified at the level of order and characterized in a frequency, dominance and constancy. The study allowed the identification of seven orders and eight categories of taxonomic taxonomy, Squamata, Diplopoda, Araneae, Coleoptera, Dermaptera, Hymenoptera, Orthoptera. It was verified the presence of greater influence of the order Hymenoptera (620 individuals) under the agroforestry system, there is no alteration in the natural conditions of the environment that characterize the presence of local individuals. In the area under soybean monoculture, the most representative example was a Coleoptera (121 individuals) which may be justified when dealing with pests in agricultural programs depending on the family. Changes in fauna characterization were observed in different systems of use with the diversity of data collected. It is concluded that the area under the agroforestry system is more static in terms of biodiversity of the fauna, when compared to agricultural areas like the monoculture of the soybean, that are affected by the fauna, in the crops, causing the alteration of the edaphic fauna.
\end{abstract}

Keywords: Soil quality; Edaphic biodiversity; Agroforestry system.
D) 0000-0002-6465-9731

Bruna de Freitas Iwata

(1) 0000-0002-0635-2651

Tancio Gutier Ailan

Costa

D) 0000-0002-8147-8019

Laécio Miranda da

Cunha

(1) 0000-0002-0979-0657

Nayara Caroline

Moreira Leopoldo

(1) 0000-0003-2029-9997

Gleide Ellen dos

Santos Clementino

(1) 0000-0001-8742-4137

Ana Carla Ribeiro

Maciel 


\section{Introdução}

A qualidade do solo está relacionada com a sua capacidade de funcionar dentro do ecossistema, visando sustentar a produtividade biológica, no qual a fauna permite manter a qualidade ambiental e promove a saúde das plantas e dos animais, sendo capazes de modificar as propriedades físicas, químicas e biológicas do solo (Baretta, 2007; Baretta et al., 2010).

Lavelle e Pashanasi (1989) salientam que comumente a outros ecossistemas naturais, uma grande porção de organismos pode habitar os solos e a abundância e diversidade dos grupos depende em geral das características dos diferentes tipos de solos, clima e sobretudo da vegetação predominante.

Pouco se conhece das funções e da importância dos organismos do solo, entretanto, é interessante destacar que a diversidade biológica do solo atua significativamente na interação com a manutenção da sua capacidade produtiva, sendo de grande importância para a decomposição e mineralização de resíduos orgânicos, favorecendo a disponibilidade de nutrientes às plantas fortalecendo o seu estado nutricional e até mesmo para outros indivíduos presentes no ambiente (Brown e Sautter, 2009).

Apesar da grande importância da fauna edáfica, tais organismos são extremamente sensíveis a modificações ocorridas no ambiente, tanto pelas características edafoclimáticas, quanto pelas práticas de manejo do solo adotadas e do tipo de cultivo utilizado, podendo afetar os organismos do solo (Baretta et al., 2011).

Pelo que aponta Nunes (2010) a fauna do solo, por apresentar alta diversidade e rápida capacidade de reprodução, é um excelente bioindicador de ambientes, e suas propriedades ou funções indicam e determinam à qualidade ou o nível de degradação do solo. Entretanto isso é rotineiramente afetado pela a redução da cobertura vegetal de áreas naturais, principalmente pelo desenvolvimento da agricultura, atrelado em muitos casos ao seu baixo nível de conservação, o que pode reduzir a diversidade dos organismos que habitam o solo e levar a extinção daqueles que são endêmicos em determinadas áreas.

Embora pouco se conheça sobre a relação dos organismos do solo e os processos biológicos, nos ecossistemas florestais e agrícolas, alguns estudos relatam a função ecológica e benefícios desses organismos para esses ecossistemas, utilizando-os como indicadores de qualidade de solo e/ou ambiental (Baretta et al., 2010; Merlin, 2005; Pereira, 2012). E neste caso, os sistemas agroflorestais (SAFs) são excelentes ambientes de estudo da fauna do solo, em virtude da semelhança com sistemas naturais (Barros et al., 2008), podendo representar a combinação ideal para a biologia do solo, ligado a oferta de refúgio $\mathrm{e}$ a alta disponibilidade de matéria orgânica, para macro e microrganismos, sem que haja grandes perturbações advindas de manejo intensivo (Luizão e Schubart, 1987).

$$
\text { Portanto, partindo do }
$$
pressuposto que as práticas agrícolas de manejo podem afetar de forma direta e indireta a fauna edáfica, o que se reflete na sua densidade e diversidade, objetivou-se com esta pesquisa avaliar a fauna edáfica em um Latossolo Vermelho-Amarelo em sistema agroflorestal recém-instalado e monocultivo de soja no Cerrado piauiense.

\section{Materiais e métodos}

\section{Área de estudo}

0 estudo foi realizado em área de Sistema Agroflorestal (SAF) recem instalado e Sistema Agrícola de Monocultivo de Soja, localizado no município de São Gonçalo do Gurgueia (Figura 1) extremo sul do Estado do Piauí. Situado a $440 \mathrm{~m}$ de altitude, a sede 
municipal tem as coordenadas a aproximadamente $820 \mathrm{~km}$ da capital do geográficas $10^{\circ} 01^{\prime} 49^{\prime \prime}$ de latitude Sul e Estado, Teresina (IBGE, 2010). $45^{\circ} 18^{\prime} 10^{\prime \prime}$ de longitude Oeste e distância

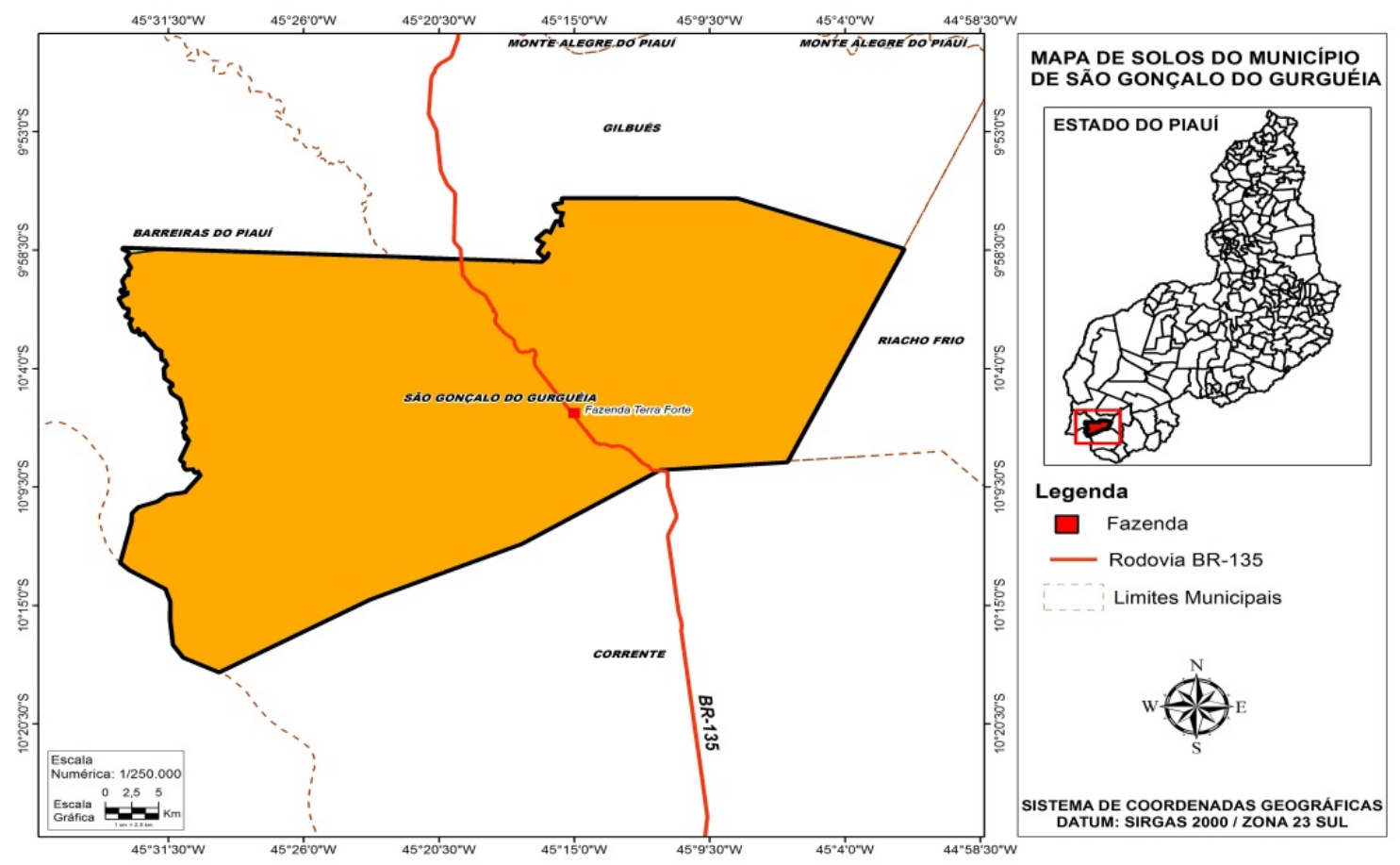

Figura 1. Localização do município de São Gonçalo do Gurgueia - Piauí. Fonte: Coelho et al., 2017.

0 município pertence à Microrregião do Alto Médio do Gurgueia, com clima tropical subúmido do tipo Aw e Bsh, conforme a classificação de Köppen, com inverno seco e verão chuvoso e precipitação média anual de 750 a $1400 \mathrm{~mm}$ e temperatura anual de $26^{\circ} \mathrm{C}$.

A fazenda agricola destinada a avaliação do estudo, se localiza a aproximadamente $10 \mathrm{~km}$ do perimetro urbano do municipio de São Gonçalo do Gurgueia.

\section{Procedimentos Metodológicos}

Para realização da pesquisa foi selecionada uma área com tamanho amostral de $10 \times 10 \mathrm{~m}$, equivalente a 0,1 ha. As armadilhas foram utilizadas para avaliar a atividade da fauna epígea, ou seja, dos componentes que atuam na superfície do solo, na área sob o SAFs e no monocultivo de soja durante o período de experimentação.

A coleta amostral da fauna edáfica realizou-se através de armadilhas de queda do tipo pitfall traps (Moldenke, 1994), constituída de recipientes plásticos com $9 \mathrm{~cm}$ de diâmetro e $11 \mathrm{~cm}$ de altura enterrados a nível do solo, contendo a solução fixadora composta por 170 a $250 \mathrm{~mL}$ de água, $100 \mathrm{~mL}$ de álcool $70 \%$ e $2 \mathrm{~mL}$ de detergente neutro.

Foram instaladas 10 armadilhas em cada área de estudo (SAF e monocultivo) com espaçamento mínimo de $1 \mathrm{~m}$ entre elas, dispostas aleatoriamente. Os espécimes coletadas foram classificadas e quantificadas quanto ao nível de ordem. 0 monitoramento das armadilhas foi realizado em um período de 60 dias em 
um ciclo de 15 dias, quantificando 4 analise amostral.

\section{Análise dos dados}

A frequência (f) das ordens das espécimes foi determinada pela participação percentual do número de indivíduos de cada ordem, em relação ao total coletado, de acordo com a fórmula:

\section{$f=n i / N \times 100$}

Em que:

$f=$ frequência relativa;

$n i=$ número total de indivíduos da ordem $i$;

$N$ = número total de indivíduos (Silveira Neto et al., 1976).

A dominância das ordens foi definida a partir da abundância relativa de cada espécie, segundo a fórmula:

\section{$D \%=i / t \times 100$}

\section{Em que:}

$D \%=$ porcentagem de dominância;

$$
i=\text { número total de indivíduos }
$$
coletados.

De acordo com as categorias estabelecidas por Friebe (1983), a dominância das ordens são consideradas conforme: eudominante $(>10 \%)$, dominante $(>5-10 \%)$, subdominante $(>2$ $5 \%)$, recessiva $(1-2 \%)$, rara $(<1 \%)$.

A constância foi calculada por meio da porcentagem de ocorrência das ordens no levantamento, utilizando-se da fórmula:

\section{$C=c i / N c \times 100$}

Em que:

$C=$ percentagem de constância;

$c i=$ número de coletas contendo a ordem $i$
$N c$ = núemero total de coletas efetuadas (Silveira Neto et al., 1976).

Pelas percentagens, as ordens foram agrupadas em categorias: ordens constantes (presentes em mais de 50\% das coletas), ordens acessórias (presentes entre 25 e $50 \%$ das coletas), ordens acidentais (presentes em menos de $25 \%$ das coletas.

\section{Resultados e discussão}

0 estudo da fauna edáfica nas duas áreas permitiram a identificação de 7 ordens, totalizando 849 indivíduos pertencente aos seguintes grupos taxonômicos: Squamata, Diplopoda, Araneae, Coleoptera, Dermaptera, Hymenoptera e Orthoptera. No entanto, durante as coletas das amostragens, foi considerado o período seco da região.

0 estudo pôde verificar que a ordem com maior representatividade dos organismos edáficos quanto a frequência na área de monocultivo foi a Coleoptera (Figura 2), no qual são abundantes nos solos do Brasil e, embora algumas familias sejam consideradas pragas em sistemas agrícolas, Silva et al. (2012) apontam que esta ordem pode contribuir para a intregridade física e química do solo.

Segundo Thomanzini e Thomanzini (2002), estes organismos atuam na incorporação e decomposição da máteria orgânica, sendo considerado por Lavelle e Spain (2001) como indicadores da qualidade do solo. Na área do SAF houve a predominância da Hymenoptera (Figura 2) na qual a cobertura vegetal pode ter favorecido um hábitat adequado a esse grupo. Sendo considerado um dos principais agentes na fragmentação da serapilheira e na incorporação de matéria orgânica no solo (Poggiani et al., 1996), e também responsáveis pela aeração do solo, aumentando a infiltração d'água e as trocas gasosas (Gassen, 1999). 


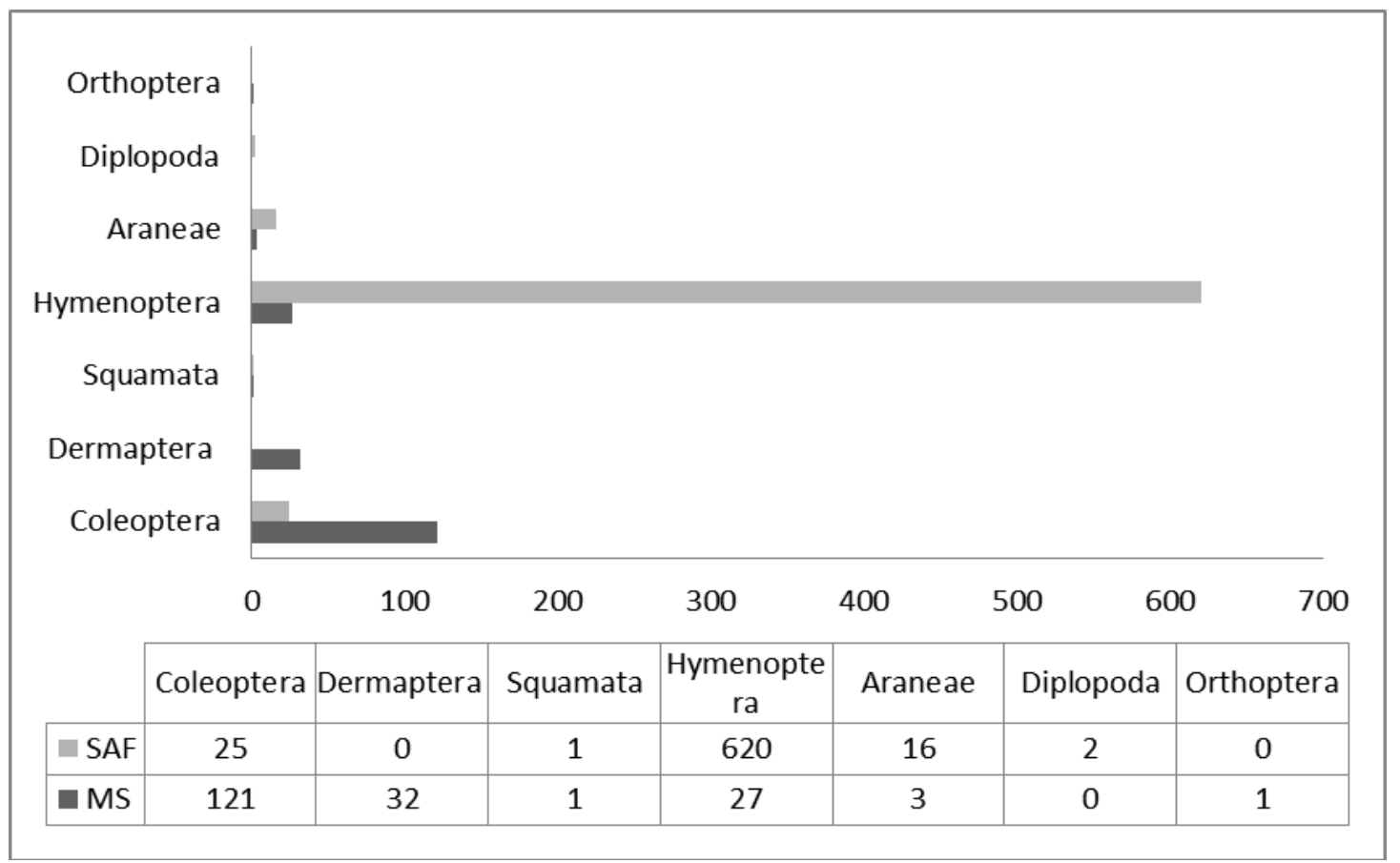

Figura 2. Número de individuos de cada ordem coletados na área do monocultivo de soja (MS) e sob o sistema agroflorestal (SAF).

As práticas de manejo utilizadas em um sistema de produção podem afetar de forma direta e indireta a fauna do solo, o que se reflete na sua densidade e diversidade. Nas áreas estudadas pôdese verificar uma diferença nas populações que foram identificadas, podendo esta sendo influenciado pela estrutura e localização de cada área. 0 SAF estudado estava associado a mata nativa e o monocultivo sob área adotada por práticas de cultivos tradicionais na agricultura.

Práticas agrícolas como o monocultivo e o preparo convencional do solo têm ocasionado alterações na biodiversidade. Uma das alternativas para a diminuição do impacto sobre a diversidade biológica do solo é o emprego de práticas agrícolas conservacionistas (Rieff, 2010).

Quanto à dominância das ordens da fauna coletada na área do monocultivo (Tabela 1), observou-se que três ordens foram caracterizadas como subdominante (Coleoptera, Dermaptera e Hymenoptera), duas denominadas como raras (Ortoptera, Squamata) e apenas uma recessiva (Araneae). Silva et el. (2015), analisando bioindicadores de qualidade edáfica em diferentes usos do solo, apontam que os grupos taxonômicos Coleoptera, Dermaptera e Hymenoptera são os melhores bioindicadores de qualidade edáfica. Alterações locais nas condições de uso e manejo, tendem a mudar o balanço competitivo entre as espécies, permitindo redistribuição na dominância entre elas (Begon et al., 1999) e, consequentemente, na estrutura das comunidades.

A dominância na área do SAF (Tabela 1), observou-se que apenas uma ordem se caracteriza como eudominante (Hymenoptera), subdominante (Coleoptera e Araneae) e raras (Squamata e Diplopoda). A dominância causada pela ordem de maior representatividade (Hymenoptera) na área do SAF se deve provavelmente pela proximidade da área com a mata nativa, 
uma vez que as formigas possuem elevada mobilidade, transitando de uma área para outra com facilidade (Araújo et al., 2010). A maior parte dos grupos de formigas não são desfolhadores, sendo consideradas de fundamental importância para a manutenção da qualidade do solo, sendo úteis como bioindicadores dessas condições (Crepaldi et al., 2014).

Tabela 1. Relação dos indivíduos quanto a constância, dominância e frequência da fauna edáfica capturadas do monocultivo de soja e no SAF.

\begin{tabular}{lcccccc}
\hline \multirow{2}{*}{ Ordem } & \multicolumn{2}{c}{ Constância* } & \multicolumn{2}{c}{ Dominância (\%) } & \multicolumn{2}{c}{ Frequência(\%) } \\
\cline { 2 - 7 } & MS & SAF & MS & SAF & MS & SAF \\
\hline Coleoptera & $100 \mathrm{x}$ & $75 \mathrm{x}$ & 65.40 & 3.75 & 65.40 & 3.75 \\
Dermaptera & $100 \mathrm{x}$ & - & 17.29 & - & 17.29 & - \\
Squamata & $25 \mathrm{y}$ & $25 \mathrm{y}$ & 0.54 & 0.15 & 0.54 & 0.15 \\
Hymenoptera & $75 \mathrm{x}$ & $100 \mathrm{x}$ & 14.59 & 93.23 & 14.59 & 93.23 \\
Araneae & $75 \mathrm{x}$ & $100 \mathrm{x}$ & 1.62 & 2.40 & 1.62 & 2.40 \\
Diplopoda & - & $50 \mathrm{x}$ & - & 0.30 & - & 0.30 \\
Ortoptera & $25 \mathrm{y}$ & - & 0.54 & - & 0.54 & - \\
\hline
\end{tabular}

*As letras após os valores representam: x - família constante; y - família acessórias. MS monocultivo de soja; SAF - sistema agroflorestal.

Em relaçao à constância das ordens amostradas (Tabela 1) no monocultivo, verificou-se que estiveram mais presentes nas coletas realizadas foram as ordens Coleoptera, Dermaptera, Hymenoptera e Araneae, sendo enquadradas como ordens constantes. Destaca-se a presença das ordens Ortoptera e Squamata como acessóriais. Para a ordem Hymenoptera, por ser abundante por ocupar os mais diversos tipos de ambientes (Alencar et al., 2007).

Em relação à Coleóptera este fato pode ser atribuído à grande diversidade de espécies e abundância, pois ocupam os mais diversos nichos ecológicos e apresentam grande diversidade de hábitos alimentares (Marinoni et al., 2001). No SAF os individuos enquadrados na ordem constantes foram Coleoptera, Hymenoptera, Araneae e Diplopoda. Apenas uma ordem, Squamata como acessórias. A um destaque para o ordem Diplopoda, presente apenas na área sob o SAF, que de acordo com Costa Neto (2007), indica que nos ambientes mais preservados, a cobertura vegetal propicia maior acúmulo de serapilheira em decomposição aumentando a população de fungos, que servem de alimentos para o grupo Diplopoda.

Alguns desses grupos, como Araneae, Hymenoptera e Coleoptera, são predadores responsáveis pela fragmentação da matéria orgânica e apresentam como importantes indicadores da qualidade do solo (Lavelle e Spain, 2001).

Os grupos taxonômicos da fauna do solo encontrados em menor número foram o Squamata, Diplopoda e Ortoptera (Figura 2). O preparo inicial realizado na área do $\mathrm{SAF}$, antes da instalação do experimento, pode ter favorecido a redução de algumas espécies menos adaptadas aos impactos provocados e a diminuição na disponibilidade de alimentos para a fauna edáfica. Quanto na área do monocultivo da soja pode ter ocorrido por influência no preparo do solo, de forma direta por danos mecânicos ou 
indireta pela compactação do solo e a ausência de cobertura do solo.

A fauna edáfica está diretamente envolvida nos processos de fragmentação da materia orgânica do solo, desempenhando papel fundamental na decomposição e na ciclagem de nutrientes. Korasaki et al., (2013) aponta que a composição de espécies da fauna do solo é função de um conjunto de fatores biológicos, que interagem entre si.

Segundo Rovedder et al. (2009), a riqueza de fauna edáfica está relacionada com a disponibilidade e qualidade de cobertura vegetal como fonte de nutrientes e abrigo. A lavração, a queimada, a exposição do solo ao sol e o uso de adubos, fazem com que a maioria da fauna desapareça (Primavesi, 1990).

Marchão et al. (2009) aponta que o revolvimento do solo e a aplicação de defensivos agrícolas podem ter efeito negativo quanto a diversidade da fauna do solo. Lima et al. (2010) trabalhando com diferentes sistemas agroflorestais, comprovaram que apresentam maior riqueza de espécies, isto porque os sistemas agroflorestais são estruturalmente mais diversos.

\section{Conclusões}

0 processo de instalação do sistema agroflorestal, provoca alterações significativas na interação de indivíduos e no manejo do solo, atuando como fator de conservação deste e consequentemente na preservação de espécies arbóreas e da fauna edáfica presente no solo.

O monocultivo de soja sofre influência direta pelas práticas adotadas nos cultivos, provocando alteração da fauna edáfica.

Embora o sistema agroflorestal esteja em etapa de implantação, observase eficiência quanto o estado de conservação da área, visto que as práticas manejadas no monocultivo de soja são eficientes favorecendo a qualidade do solo.

\section{Conflito de interesses}

Os autores declaram não haver conflito de interesses.

\section{Referências}

Alencar, I. D. C. C.; Fraga, F. B.; Tavares M. T.; Azevedo, C. O. Perfil da fauna de vespas parasitóides (Insecta, Hymenoptera) em uma área de Mata Atlântica do Parque Estadual de Pedra Azul, Domingos Martins, Espírito Santo, Brasil. Arquivos do Instituto Biológico, v. 74, n. 2, p. 111-114, 2007.

Araújo, L. H. A.; Souza, C.; Oliveira, S. J. C.; Souto, S. J.; Soares, J. J. Macrofauna edáfica sob diferentes ambientes em Latossolo da Região Agreste. Anais do IV Congresso Brasileiro de Mamona e I Simpósio Internacional de Oleaginosas Energéticas, João Pessoa, CBM, 2010.

Baretta, D.; Santos, J. C. P.; Sehat, C. J.; Geremia, V. E.; Oliveira Filho, L. C. I.; Alves, M. V. Fauna edáfica e qualidade do solo. Tópicos em Ciências do Solo, v. 7, p. 119-170, 2011.

Barros, E.; Mahieu, J.; Tapia-Coral, S.; Nascimento, A. R. L.; Lavelle, P. Comunidade da macrofauna do solo na Amazônia brasileira. In: Moreira, F. M. S.; Siqueira, J. O.; Brussaard, L. (Ed.). Biodiversidade do solo em ecossistemas brasileiros. Lavras: UFLA, 2008. p. 171-191.

Begon, M.; Harper, J. L.; Townsend, C. R. Ecology. Berlin: Blackwell, 1999.

Costa Neto, E. M. The perception of Diplopoda (Arthropoda, Myriapoda) by the inhabitants of the County of Pedra Branca, Santa Teresinha, Bahia, Brazil. Acta Biólogica Colombiana, v. 12, n. 2, p. 123-134, 2007. Disponível em: <http://www.scielo.org.co/ scielo.php?script=sci_arttext\&pid=S0120-

548X2007000200010>. Acesso em: 23 abr. 2018.

Crepaldi, R. A.; Portilho, I. I. R.; Silvestre, R.; Mercante, F. M. Formigas como bioindicadores da qualidade do solo em sistema integrado lavoura-pecuária. Ciência Rural, $\quad$ v. 44, p. 781-787, 2014. https://doi.org/10.1590/S0103-84782014 000500004

Friebe, B. Zür Biologie eines Buchenwald bodens: 3 . Die Kaferfauna. Karlshue, Carolinea, v. 41, p. 45-80, 1983. 
Gassen, D. N. Os insetos e a fertilidade do solo. Resumos do Curso sobre Aspectos Básicos de Fertilidade e Microbiologia do Solo sob Plantio Direto, Cruz Alta, 1999. p. 70-89.

IBGE - Instituto Brasileiro de Geografia e Estatística. Rio de Janeiro, 2010.

Korasaki, V.; Morais, J. W.; Braga, R. F. Macrofauna. In: Moreira. F. M. S.; Cares, J. E.; Zanetti, R.; Stürmer, S. L. O ecossistema solo: componentes, relações ecológicas e efeitos na produção vegetal. Lavras: Editora da UFLA, 2013. p. 79-128.

Lavelle, P.; Pashanasi, B. Soil macrofauna and land management in Peruvian Amazonia (Yurimaguas, Loreto). Pedobiologia, v. 33, p. 283-290, 1989.

Lavelle, P.; Spain, A. V. Soil Ecology. Dordrecht: Kluwer Academic, 2001.

Lima, S. S.; Aquino, A. M.; Leite, L. F. C.; Velásquez, E.; Lavelle, P. Relação entre macrofauna edáfica e atributos químicos do solo em diferentes agroecossistemas. Pesquisa Agropecuária Brasileira, v. 45, n. 3, p 322-331, 2010. https://doi.org/ 10.1590/S0100-204X2010000300013

Luizão, F. J.; Schubart, H. O. R. Litter production and decomposition in a terrafirme forest of Central Amazonia. Experientia, v. 43, n. 3, p. 259-265, 1987. https://doi.org/10.1007/BF01945549

Marchão, R. L.; Lavelle, P.; Celini, L.; Balbino, L. C.; Vilela, L. Soil macrofauna under integrated crop-livestock systems in a Brazilian Cerrado. Pesquisa Agropecuária Brasileira, v. 44, n. 8, p. 1011-1020, 2009. https://doi.org/10.1590/S0100-204X2009 000800033

Marinoni, R. C.; Ganho, N. G.; Monné, M. L.; Mermudes, J. R. M. Hábitos alimentares em Coleoptera (Insecta). Ribeirão Preto: Holos, 2001.

Moldenke, A. R.; Arthropods. In: Weaver, R. W.; Angle, S.; Bottomley, P.; Bezdicek, D.; Smith, S.; Tabatabai, A.; Wollum, A. Methods of soil analysis: Microbiological and biochemical properties. Madison: SSSA, 1994. Part 2. p. 517-542.
Poggiani, F.; Oliveira, R. E.; Cunha, G. C. Práticas de Ecologia Florestal. Documentos Florestais, n. 16, p. 1-44, 1996.

Primavesi, A. Manejo ecológico do solo: agricultura em regiões tropicais. 9. ed. São Paulo: Nobel, 1990.

Rieff, G. G. Monitoramento de ácaros e colêmbolos como indicadores de qualidade do solo. Porto Alegre: Faculdade de Agronomia, Universidade Federal do Rio Grande do Sul, 2010. (59 p. Dissertação de mestrado em Ciência do Solo).

Rovedder, A. P. M.; Eltz, F. L. F.; Drescher, M. S.; Schenato, R. B.; Antoniolli, Z. I. Organismos edáficos como bioindicadores da recuperação de solos degradados por arenização no Bioma Pampa. Ciência Rural, v. 39, n. 4, p. 1061-1068, 2009. https://doi.org/ 10.1590/S0103-84782009005000023

Silva, D. A. A.; Silva, D. M.; Jacques, R. J. S.; Antoniolli, Z. I. Bioindicadores de qualidade edáfica em diferentes usos do solo. Enciclopédia Biosfera, v. 11, p. 3728, 2015. Disponível em: <http://www.conhecer. org.br/enciclop/2015c/agrarias/bioindicado res $\% 20 d e \% 20$ qualidade.pdf $>$. Acesso em: 23 abr. 2018.

Silva, R. F.; Scheid, D. L.; Corassa, G. M.; Bertollo, G. M.; Kuss, C. C.; Lamego, F. P. Influência da aplicação de herbicidas préemergentes na fauna do solo em sistema convencional em plantio de cana-de-açúcar. Biotemas, v. 25, n. 3, p. 227-238, 2012. https://doi.org/10.5007/2175-7925.2012 v25n3p227

Silveira Neto, S.; Nakano, O.; Vila Nova, N. A. Manual de ecologia dos insetos. Piracicaba: Ceres, 1976.

Thomanzini, M. J.; Thomanzini, A. P. B. W. Levantamento de insetos $\mathrm{e}$ análise entomofauna em florestas, capoeira e pastagem no Sudeste Acreano. Rio Branco: Embrapa Acre, 2002. 